\title{
The role of surgery in Conn's syndrome - a case of refractory hypertension secondary to an aldosterone secreting adenoma
}

\author{
MD Carides, ${ }^{\text {(D) NT Sishuba, }}{ }^{2}$ (D) I Bombil, ${ }^{3}$ (D) C Christofides $^{3}$ iD \\ ${ }^{1}$ Medical Outpatient Department, Bertha Gxowa Hospital, South Africa \\ ${ }^{2}$ Department of Surgery, Charlotte Maxeke Johannesburg Academic Hospital, South Africa \\ ${ }^{3}$ Department of Surgery, Chris Hani Baragwanath Academic Hospital, South Africa
}

Corresponding author, email: michaelac@live.co.za

\begin{abstract}
Summary
Primary aldosteronism, eponymously known as Conn's syndrome, has recently gained recognition as the most common cause of endocrine hypertension. Unilateral subtypes, such as aldosterone secreting adenomas, are managed surgically and are potentially curable. The background of refractory hypertension and hypokalaemia in a forty-year-old man raised suspicions of Conn's syndrome which was localised to an aldosterone secreting adenoma in the right adrenal gland. The patient underwent a laparoscopic intraperitoneal right adrenalectomy which resulted in normalisation of his plasma aldosterone concentration as well as improved blood pressure control.
\end{abstract}

\section{Case presentation}

A forty-year-old man with no known comorbidities presented to the emergency department with a two-day history of left upper limb paraesthesia. He had sober habits and no significant family medical history. On objective examination, he had a blood pressure of $210 / 150$, normal BMI and no signs of neurological fallout. He was admitted as a hypertensive emergency for blood pressure control and inpatient workup.

Baseline investigations identified target organ damage with left ventricular hypertrophy on ECG and renal dysfunction with a serum urea and creatinine of $9.3 \mathrm{mmol} / \mathrm{L}$ (normal range 2.1-7.1) and $161 \mathrm{umol} / \mathrm{L}$ (normal rage 64-104) respectively. Abdominal ultrasound for suspected chronic kidney disease showed a right adrenal mass. He was initiated on antihypertensive treatment, booked for contrast abdominal CT scan and discharged for outpatient management.

On follow-up visit, he had a blood pressure of 171/110 and no systemic complaints. Counselling confirmed he had been adherent on four antihypertensive agents and was following dietary advice regarding salt restriction. Repeat biochemical investigations showed renal dysfunction had resolved and a hypokalaemia of $2.6 \mathrm{mmol} / \mathrm{L}$ (normal range 3.5-5.1).

The background of resistant hypertension and hypokalaemia raised suspicions of secondary hypertension as a result of primary aldosteronism (PA). Screening tests showed a suppressed upright plasma renin activity (PRA) of $<0.5$ $\mathrm{mIU} / \mathrm{L}$ (normal range 4.4-46.1) and a markedly elevated upright plasma aldosterone concentration (PAC) of 2290 pmol/L (normal range 111-859). The absolute aldosterone: renin ratio (ARR) could not be calculated as PRA was below assay detection limits. Testing was carried out while the patient was on a regimen of amlodipine, lasix and atenolol. Of note, beta blockers and aldosterone antagonists lead to an increase in the ARR, resulting in false positive results during screening.

Confirmatory tests, such as the oral salt loading test, saline infusion test, fludrocortisone suppression test and captopril challenge, to verify PA were not indicated. ${ }^{1}$ Confirmatory testing is usually considered mandatory with an exception in cases of PA with spontaneous hypokalaemia, a PAC greater than $550 \mathrm{pmol} / \mathrm{L}$ and a PRA below assay detection limits. ${ }^{1}$

Adrenal protocol contrast CT scan showed a right adrenal mass measuring $21.8 \times 21.0 \times 21.8 \mathrm{~mm}$ in size with a radiodensity of $95.9 \mathrm{HU}$ on the portovenous phase. The mass had an absolute washout of $80.5 \%$ and a relative washout of $55.8 \%$ in keeping with an adrenal adenoma. Aside from the adrenal adenoma, the right adrenal gland had normal morphology and parenchymal enhancement. The left adrenal gland was normal in size with no evidence of cysts or masses. The limbs of both adrenal glands were not enlarged, lowering suspicions of bilateral adrenal hyperplasia.

Adrenal vein sampling (AVS) which is the gold standard investigation to lateralise the source of aldosterone production was not utilised. It is a technically challenging procedure with high failure rates.,3 Furthermore, a lack of standardisation for interpreting results and different thresholds for determining unilateral from bilateral hyperaldosteronism detract from its practicality. ${ }^{2,3}$ As a result, Endocrine Society guidelines suggest that AVS can be avoided in young patients with severe PA and a CT scan showing a unilateral adenoma with a normal contralateral adrenal gland. ${ }^{3}$

Endocrine consultation was done as soon as PA was suspected to assist with the patient's workup and management. Despite optimisation using six antihypertensive agents 


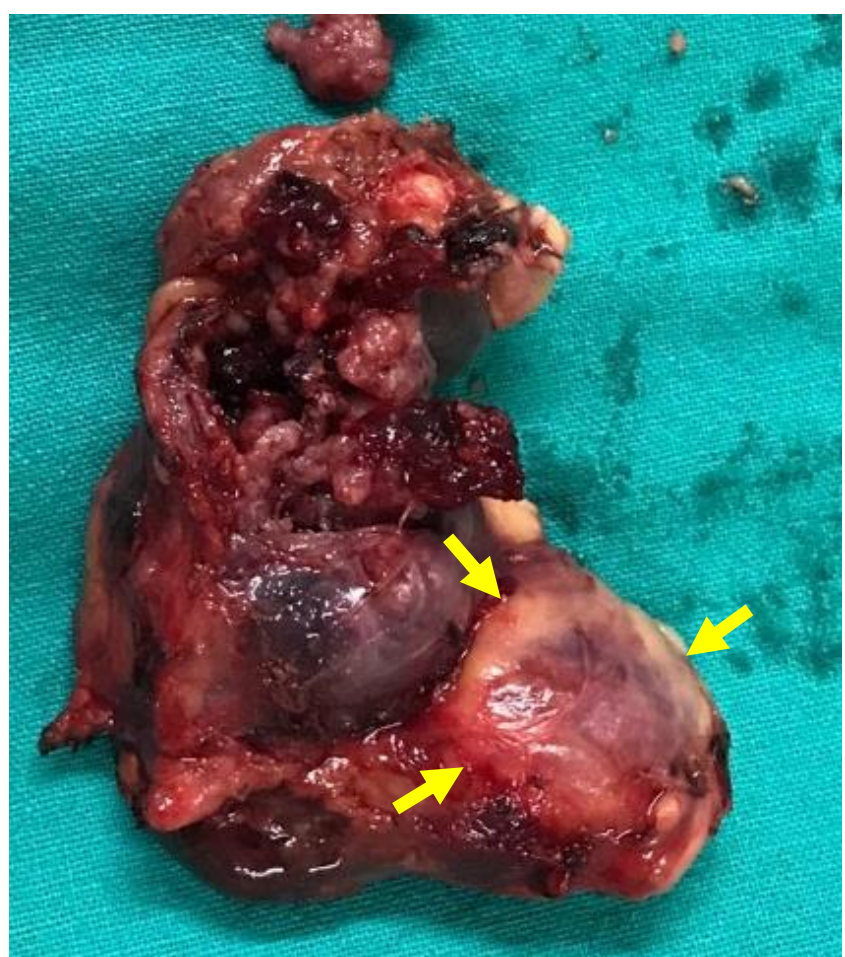

Figure 1: Arrows identifying the adenoma measuring $25 \times 22 \times 20 \mathrm{~mm}$ on the surface of the right adrenal gland

(mineralocorticoid receptor antagonists included) and strict sodium restriction, the patient's blood pressure remained uncontrolled.

A multidisciplinary team was utilised throughout which comprised of an endocrinologist, dietician, chemical pathologist, radiologist and the surgical team. The diagnosis of PA secondary to an aldosterone secreting adenoma (ASA) was made. Multidisciplinary meetings and discussion with the patient concluded that unilateral adrenalectomy was indicated.

The patient underwent an uneventful laparoscopic intraperitoneal right adrenalectomy. Histopathological report confirmed an adrenal nodule measuring $25 \times 22 \times 20 \mathrm{~mm}$ in keeping with an adenoma. At his six-week follow-up visit, the hypokalaemia had resolved, PAC had normalised to 26.9 $\mathrm{pmol} / \mathrm{L}$ and his blood pressure was well controlled on two agents.

\section{Discussion}

Conn's syndrome was first described by Jerome W Conn who in 1955 published the first report on PA in medical literature. ${ }^{4}$ Hypertension secondary to $\mathrm{PA}$ is rare; most cases are of essential hypertension, and therefore current international hypertensive practice guidelines do not recommend the routine screening of all hypertensive patients. ${ }^{5}$ Guidelines do, however, maintain that clinicians should have a high index of suspicion and screen for PA in cases of refractory hypertension. The incidence of PA was previously underestimated in the hypertensive population and it has now emerged as the leading cause of endocrine hypertension. ${ }^{5}$

PA typically presents with refractory hypertension and hypo- or normokaleamia. ${ }^{5}$ There are two major subtypes of PA. ASA being the most common form of the unilateral subtype and idiopathic hyperaldosteronism (IHA) being the most common form of the bilateral subtype. ${ }^{6}$ When the

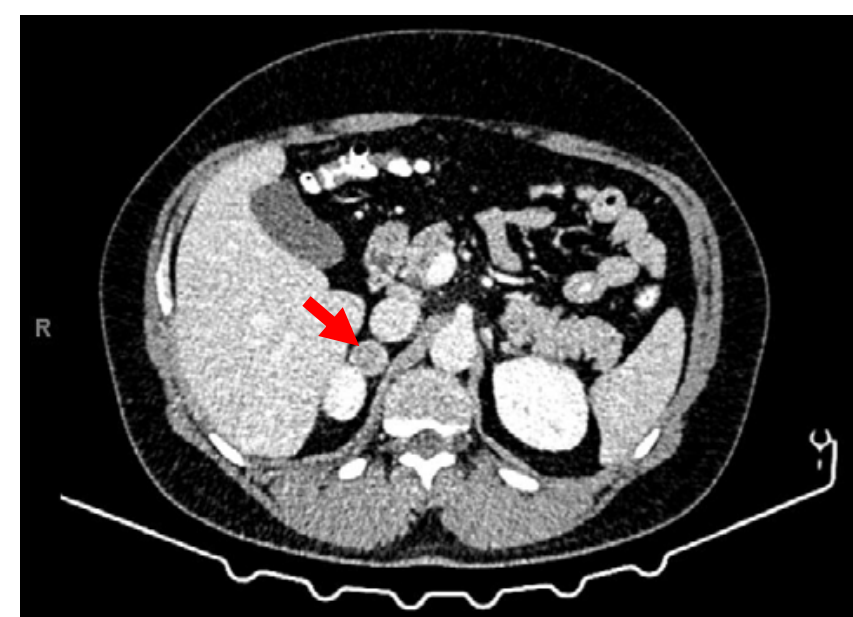

Figure 2: Adrenal protocol CT scan showing the right adrenal adenoma on the portovenous phase

general population is screened for PA, IHA accounts for $60-66 \%$ of cases and ASA accounts for $30-35 \%$ of cases. ${ }^{7}$ Proper distinction between IHA and ASA is crucial as the former is treated medically with mineralocorticoid receptor antagonist therapy and the latter surgically, by unilateral adrenalectomy, with the potential for cure. ${ }^{8}$

PA patients with unilateral disease treated exclusively with mineralocorticoid receptor antagonist therapy had a substantially higher risk of cardiovascular events and hypertensive target organ damage compared to those treated with adrenalectomy. ${ }^{9}$ This emphasises the importance of screening and early referral for surgical management. Unfortunately, a lack of awareness and understanding of PA and its workup presents a barrier to surgical referral.

Another barrier to the surgical management of PA is difficulty differentiating bilateral from unilateral disease owing to the absence of an accessible, non-invasive and accurate localisation test. The use of CT and MRI produces low-resolution images which are unable to detect aldosterone-producing microadenomas., ${ }^{2,3}$ Furthermore, non-functioning adrenal adenomas/incidentalomas (which are not uncommon in the older population) may be present on imaging. ${ }^{2,3}$ These are irrelevant and may distract the practitioner from the true underlying pathology.

Unilateral adrenalectomy presents the possibility for complete clinical success (resolution of hypertension) and complete biochemical success (resolution of PA and hypokalaemia). Various predictive models of aldosteronomaassociated hypertension resolution after adrenalectomy exist. The four-item aldosteronoma resolution score (ARS) using the regression coefficients of the Utsumi et al. (2014) Japanese model has been shown to have superior accuracy. ${ }^{10}$ Four preoperative clinical parameters including BMI, sex, duration of hypertension and number of preoperative antihypertensive medications are taken into account to calculate the ARS. ${ }^{11}$

The use of predictive models, especially when there is low likelihood of biochemical/clinical success, and the diagnostic difficulty presented by localising unilateral PA should not be a deterrent to surgery. Surgical intervention has shown to substantially reduce the risk of cardiovascular events and hypertensive target organ damage and thus plays a major role in the multidisciplinary management of PA secondary to an ASA. ${ }^{9}$ 


\section{Conflict of interest}

The authors declare no conflict of interest.

\section{Funding source}

None.

\section{Ethical approval}

Ethics committee approval was received from the University of the Witwatersrand Human Research Ethics Committee R14/49.

\section{ORCID}

MD Carides (iD https://orcid.org/0000-0003-1428-9493

NT Sishuba (iD https://orcid.org/0000-0003-2333-8867

I Bombil (iD) https://orcid.org/0000-0002-4819-0785

C Christofides (iD https://orcid.org/0000-0003-0330-2963

\section{REFERENCES}

1. Funder JW. The management of primary aldosteronism: case detection, diagnosis, and treatment: an endocrine society clinical practice guideline. J Clin Endocrinol Metab. 2016;101:1889-916. https://doi.org/10.1210/jc.2015-4061.

2. Deipolyi AR. Adrenal vein sampling for Conn's syndrome: diagnosis and clinical outcomes. Diagnostics. 2015;5:254-71. https://doi.org/10.3390/diagnostics5020254.

3. Rossi GP. An expert consensus statement on use of adrenal vein sampling for the subtyping of primary aldosteronism. Hypertension. 2014;63:151-60. https://doi.org/10.1161/ hypertensionaha.113.02097.
4. Conn JW. Part I. Presidential address. I. Painting background. II. Primary aldosteronism, a new clinical syndrome. J Lab Clin Med. 1955 Jan;45(1):3-17. https://doi.org/10.5555/ uri:pii:0022214355900482.

5. Thomas RM. Endocrine hypertension: an overview on the current etiopathogenesis and management options. World J Hypertens. 2015;5:14-27. https://doi.org/10.5494/wjh.v5.i2. 14.

6. Rossi GP. Primary aldosteronism: Part II: subtype differentiation and treatment. J Nephrol. 2008;21:455-62.

7. Lee FT, Elaraj D. Evaluation and management of primary hyperaldosteronism. Surg Clin North Am. 2019;99(4):731-45. https://doi.org/10.1016/j.suc.2019.04.010.

8. Williams TA. Diagnosis and management of primary aldosteronism: the Endocrine Society guideline 2016 revisited. Eur J Endocrinol. 2018;179(1):R19-R29. https:// doi.org/10.1530/EJE-17-0990.

9. Hundemer GL. Management of endocrine disease: the role of surgical adrenalectomy in primary aldosteronism. Eur J Endocrinol. 2020;183:R185-R196. https://doi.org/10.1530/ EJE-20-0863.

10. Thiesmeyer JW. Hypertension resolution after adrenalectomy for primary hyperaldosteronism: which is the best predictive model? Surgery. 2020;169:133-7. https://doi.org/10.1016/j. surg.2020.04.017.

11. Zarnegar R, Young WF Jr, Lee J. The aldosteronoma resolution score: predicting complete resolution of hypertension after adrenalectomy for aldosteronoma. Ann Surg. 2008;247(3):5118. https://doi.org/10.1097/SLA.0b013e318165c075. 